\title{
DVB-S based Passive Radar for Short Range Security Application
}

\author{
Francesca Filippini, Octavio Cabrera, Carlo Bongioanni, Fabiola Colone, Pierfrancesco Lombardo \\ Dept. of Information Engineering, Electronics and Telecommunications (DIET) \\ Sapienza University of Rome, Via Eudossiana 18, 00184 Rome, Italy \\ \{francesca.filippini, octavio.cabrera, carlo.bongioanni, fabiola.colone, pierfrancesco.lombardo\}@uniroma1.it
}

\begin{abstract}
DVB-S based passive radars have been shown to effectively detect small RCS targets in the short-range, providing high range and velocity resolution. However, the critical infrastructure protection application also requires: (i) an adequate angular measurement accuracy, to localize to targets in the Cartesian Plane, and (ii) a real-time processing capability, able to provide prompt and frequently updated monitoring. This work presents an architecture and a processing scheme able to provide these characteristics and it demonstrates it by fielding, within Sapienza University of Rome, a low-cost COTS-based PCL receiver. The analysis of the experimental results validates the selected solution and demonstrates its high effectiveness.
\end{abstract}

Keywords- Passive Radar, DVB-S signals, short-range surveillance, direction of arrival estimation.

\section{INTRODUCTION}

The development of effective and low-cost surveillance sensors to detect small-size intruding vehicles approaching critical infrastructures finds its place among the major challenges of the last decades. In fact, providing high level of security and protection of the critical infrastructures, such as airports and harbors as well as train stations, stadiums, and even specific buildings, potentially threatened by small-size vehicles, motivated recently significant research activities.

Passive radar, also known as passive coherent location (PCL) systems, can provide an attractive solution to these problems, [1]-[3]. The absence of emissions makes them particularly suited for highly populated scenarios and does not require governmental authorizations, not to mention that concealed operation makes them difficult to be identified by potential threats. The use of PCL systems in short range surveillance applications has been investigated in several works, exploiting different Illuminators of Opportunity (IOs), such as terrestrial digital video and audio broadcast services [4]- [8], GSM signals [9] and WiFi transmissions [10], as well as satellite transmitters like the Global Navigation Satellite System [10][11]-[12] and the Digital Video Broadcasting Satellite (DVB-S) [13]-[15]. The referenced papers have demonstrated that PCL sensors can be effectively employed to detect small radar cross section (RCS) targets in short range applications. Among the different considered IOs, DVB-S waveforms were shown to be particularly suited for the short range surveillance required by infrastructure

This work has been partially supported by MIUR (Italian Ministry of Education, University and Research) under the project, "S4E Sistemi di sicurezza e protezione per l'Ambiente Mare" identification code SCN 00393 protection application [13]. This is easily recognized when considering that even a single DVB-S channel typically has a frequency bandwidth of approx. $30 \mathrm{MHz}$, allowing a suitable range resolution of about $10 \mathrm{~m}$. In addition, DVB-S satellites operate in the Ku band, namely with wavelengths in the order of few centimeters, potentially enabling at the same time also a high velocity resolution. Finally, the wide coverage of vast surface areas makes them appealing also in areas where terrestrial transmitters have poor coverage or are not reliably available. Despite the low power of the received signals, the first results reported in [13] show that in the short range of the receiver, DVB-S based PCL systems are able to detect even targets characterized by low RCS values, as cars, humans and drones.

However, to fully enable the use of these sensors in high resolution, short-range surveillance applications, two key requirements must be met:

(i) the sensor must provide an angular measurement capability with an accuracy compatible with the available range and velocity accuracies, so that a reliable localization of the targets is possible in the Cartesian plane,

(ii) the sensor must operate in real-time, providing prompt and frequently updated results to operate effectively for the short-range protection.

The purpose of this work is to demonstrate that a low-cost sensor can be obtained, which provides the two required features above, namely a capability to accurately estimate the direction of arrival (DoA) of the target echo by employing a very simple and low complexity signal processing that enables achieving real-time operations with a reasonable computational load. To this purpose, we present a 4-channel DVB-S based PCL architecture exploiting three nonuniformly spaced surveillance receiving antennas together with a high gain reference antenna to collect the direct signal. A prototypal implementation of this concept was developed at Sapienza University of Rome, which only includes commercial off the shelf (COTS) components, together with the fast processing schemes. The analysis of the real data collected by the prototype demonstrate the effectiveness of the adopted architecture and processing strategies.

The paper is organized as follows. In Section II, we describe the low-cost system architecture and the employed low-cost processing, together with the rationale behind the presented solutions. In Section III we describe the developed experimental setup and the acquisition campaign while Section IV reports some experimental results. Finally, our conclusions are reported in Section V. 


\section{LOW COST DVB-S BASED PCL SYSTEM AND EMPLOYED SIGNAL PROCESSING}

The developed DVB-S based PCL signal processing architecture is shown in Fig. 1. The first stage is the RangeVelocity map evaluation, separately performed at each surveillance channel. It is well known that the first stage of PCL processing is usually the disturbance cancellation [15], aimed at removing the direct signal contribution at the surveillance antennas. However, based on the typical direct signal to noise (DNR) and target signal to noise ratio (SNR) values measured at the surveillance antennas for the DVB-S based PCL, this stage does not appear strictly necessary. In fact, a target echo outside the zero-Doppler filter, after coherent integration along a split second, is typically well above the direct signal side lobes, which are buried below the noise floor. This is confirmed by the analysis in Section IV. Therefore, we operate without a disturbance cancellation stage. To further reduce system complexity and computational cost, the range-velocity map is evaluated using a sub-optimal batches approach [17]. Specifically, a Coherent Processing Interval (CPI) of received surveillance $\mathbf{s}$ and reference signal $\mathbf{r}$ are subdivided into $N_{\mathrm{B}}$ batches of $L_{\mathrm{B}}$ samples each (with $L_{B} N_{B}=N_{C P I}$ ) and the generic range and Doppler bin $[l, m]$ of the cross-ambiguity function (CAF) is evaluated as follows:

$$
\begin{array}{r}
\chi[l, m]=\sum_{n=0}^{N_{B}-1} \sum_{q=0}^{L_{B}-1} s\left[n L_{B}+q\right] r^{*}\left[n L_{B}+q-l\right] \\
\times \exp \left[-j 2 \pi \frac{m\left(n L_{B}+q\right)}{N_{C P I}}\right]
\end{array}
$$

where indeces $n$ and $q$ indicate respectively slow- and fasttime domain. The SNR loss introduced using the latter with respect to an optimum algorithm for CAF evaluation can be controlled by properly selecting the batches length $L_{\mathrm{B}}$ [17]. In the analyses reported in this paper, we force the SNR loss to be $0.1 \mathrm{~dB}$. Once a range-velocity map has been obtained for each surveillance channel, a cell average constant false alarm rate (CA-CFAR) detection strategy is applied. After a $K$-outof- $K$ detection criterion applied over the $K$ surveillance channels, a nominal $P_{f a}=10^{-6}$ is obtained for the detection in the whole range-velocity plane. Finally, for each rangevelocity bin that exceeds the detection threshold, the DoA $\phi_{0}$ is estimated by applying a maximum likelihood (ML) strategy, [18]:

$$
\widehat{\phi}_{0}=\underset{\phi}{\operatorname{argmax}}\{V(\phi)\}=\underset{\phi}{\operatorname{argmax}}\left\{\left|\mathbf{s}^{H}(\phi) \mathbf{x}\right|^{2}\right\}
$$

being $\mathbf{x}$ the $K \times 1$ vector that contains the complex values extracted from the $K$ range-velocity maps at the location where a detection was declared.

We assume vector $\mathbf{x}$ to be composed by both target and noise contributions, as

$$
\mathbf{x}=A \mathbf{s}\left(\phi_{0}\right)+\boldsymbol{n}
$$

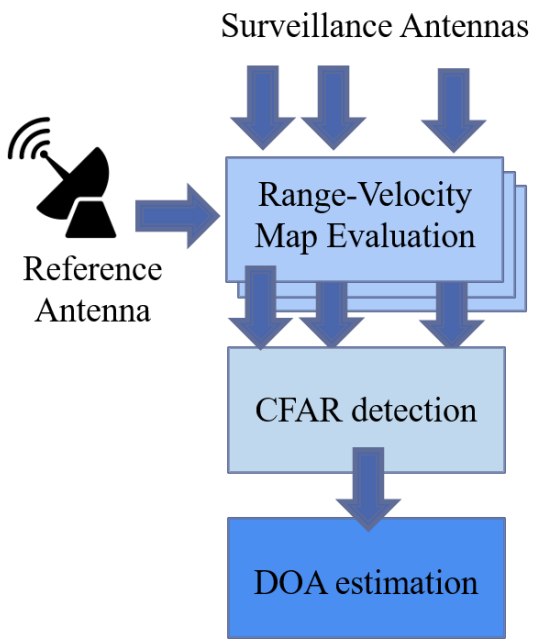

Fig. 1 Employed PCL processing scheme.

where $A$ denotes the unknown deterministic complex target amplitude while $\mathbf{s}\left(\phi_{0}\right)$ is the target steering vector and accounts for the array response from the target DoA. For a linear array composed by $K$ elements at positions $\mathbf{d}=$ $\left[\begin{array}{llll}0 & d_{1} & \ldots & d_{K-1}\end{array}\right]$ measured with respect to a first array element, $\mathbf{s}\left(\phi_{0}\right)$ can be written as

$$
\mathbf{s}\left(\phi_{0}\right)=\left[1 \quad e^{\left(-j \frac{2 \pi}{\lambda} d_{1} \sin \left(\phi_{0}\right)\right)} \quad \cdots e^{\left.\left(-j \frac{2 \pi}{\lambda} d_{K-1} \sin \left(\phi_{0}\right)\right)\right]^{H}}\right.
$$

where $\lambda$ is the carrier wavelength and $\mathbf{n}$ a $K \times 1$ vector that contains the noise samples at the surveillance channels. Under the assumption of negligible direct signal contribution in the map outside of the zero Doppler bin, we assume that $\mathbf{n}$ is a white zero-mean complex Gaussian random variable, with mean square value $\sigma_{n}^{2}$.

It is well known that depending on the number of receiving antennas and on their relative distance, the angular sector where the DoA can be unambiguously estimated using (2) as well as the achievable estimation accuracy change. In the attempt of achieving good localization results with a lowcost PCL system, two surveillance antennas could be exploited if their relative distance could be arbitrarily set. In practice, however, even if the angular sector of interest is not large $\left(30^{\circ}\right)$, it might not be possible to install the antennas as close as needed to achieve a non-ambiguous DoA estimation. In the presented experimental setup (details in Section III), the minimum distance between two antennas was $0.1 \mathrm{~m}$, which corresponds to approx. 4 wavelengths for a signal in the Ku band. Fig. 2 reports the normalized likelihood $V(\phi)$ defined as in (2) for the different array configurations considered in the following, for a target at $\phi_{0}=0^{\circ}$ in a noisefree scenario.

Specifically,

(i) when $K=2$, a good estimation accuracy must be traded with a narrow unambiguous angular sector, and vice versa. In fact, the larger is the distance between the two antennas, the narrower is the main lobe of the likelihood function but the closer the grating lobes get to the main lobe (see e.g. the comparison between light blue and yellow curves in Fig. 2). 


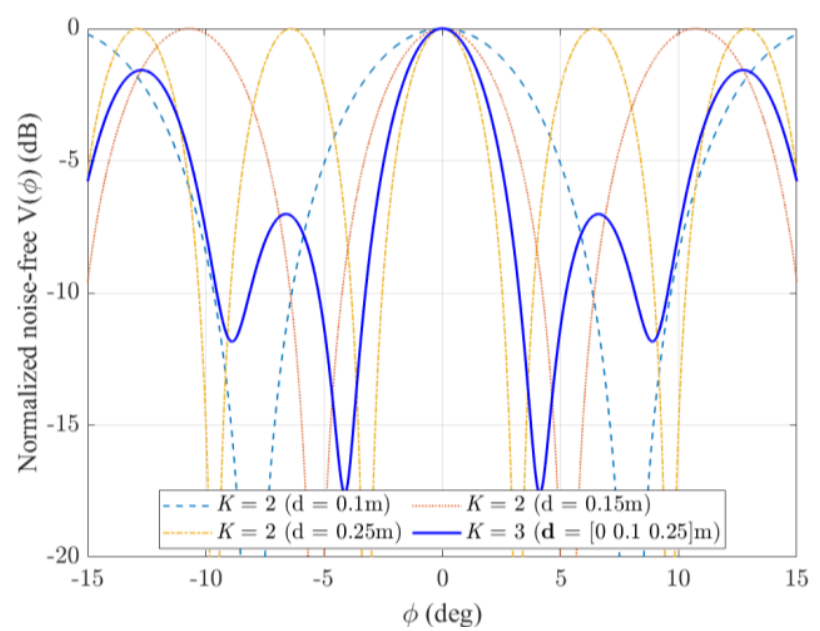

Fig. 2 Normalized noise-free $V(\phi)$ for different configurations of $K=2$ or $K=3$ surveillance antennas.

(ii) The trade-off between the DoA estimation accuracy and unambiguous angular sector can be relaxed using a $K=3$ non-uniform linear array (NULA) configuration with properly selected distances among the antennas [19]. In Fig. 2 (see the continuous blue curve), the selected NULA $\left(\mathbf{d}=\left[\begin{array}{lll}0 & 0.1 & 0.25\end{array}\right] \mathrm{m}\right)$ configuration allows us to guarantee the absence of deterministic ambiguous estimation errors in the angular sector of interest, while maintaining a good estimation accuracy. The only possible ambiguous estimates are due to the statistical occurrence of outliers, that strictly depend on the SNR of the target echo. Their occurrence has been analytically characterized in the socalled threshold region [20][21] and this theorical result can be used to optimize the sensors.

The following Section will be devoted to the description of the developed experimental setup and of the acquisition campaign that has been carried out.

\section{DEVELOPED EXPERIMENTAL SETUP AND ACQUISITION CAMPAIGN}

The main components of the COTS based passive radar demonstrator developed at Sapienza are summarized in Table I. Fig. 3(a) shows a block diagram of the system architecture. As described in section II and shown in Fig. 3(b), three feedhorn antennas are installed, placed so that their phase centers are at distances $d=\left[\begin{array}{lll}0 & 0.1 & 0.25\end{array}\right] \mathrm{m}$. A parabolic reflector (see Fig. 3(c)) is used as reference antenna, with the same feedhorn used for the surveillance antennas, located in the focus of the parabola. Specifically, the reference antenna has a beamwidth minor than $3^{\circ}$ and a gain of approx. $40 \mathrm{~dB}$, while each surveillance antenna has a beamwidth of approx. $60^{\circ}$ and a gain of approx. $12 \mathrm{~dB}$.

TABLE I EMPLOYED COMPONENTS FOR THE RF FRONT-END.

\begin{tabular}{ll}
\hline Component & Model \\
\hline Parabolic reflector & SKYWARE 96 cm \\
Feed & SKYWARE for 96 cm antenna \\
Acquisition System & NI USRP-2955 \\
LNB & SMW Ku Band LNB \\
Diplexer & SMW diplexer with 10 MHz ref. input \\
\hline
\end{tabular}

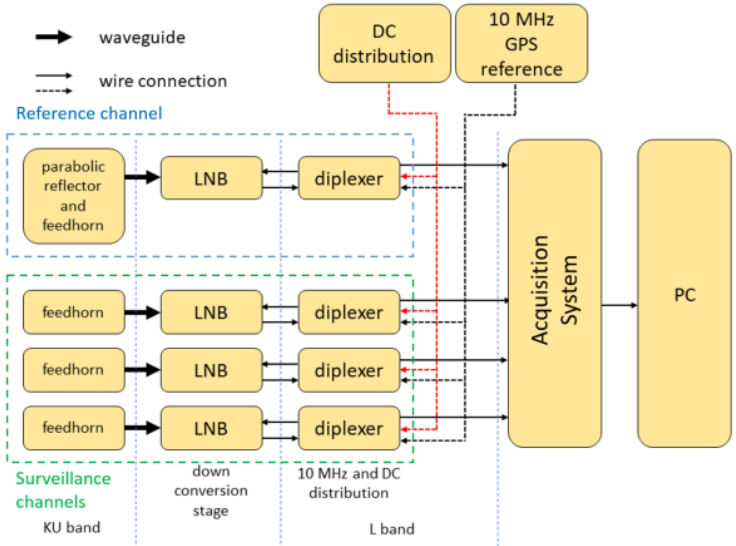

(a)

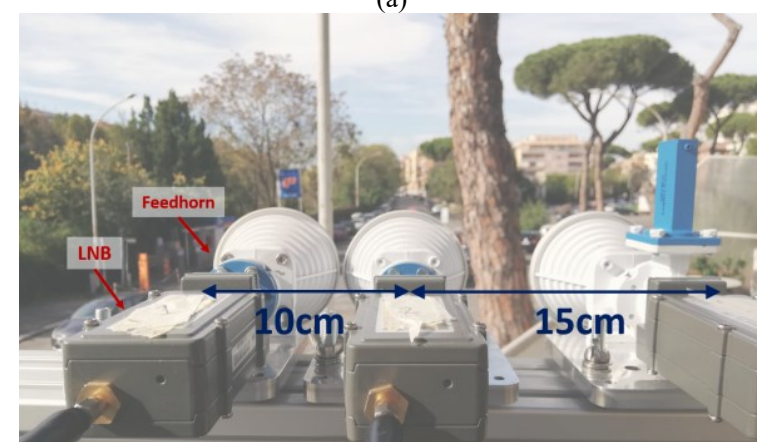

(b)

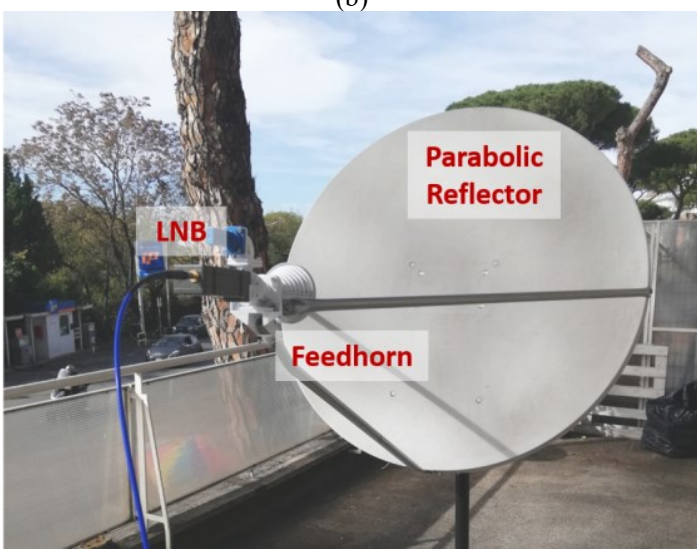

(c)

Fig. 3 Experimental setup

(a) Block diagram of the RF front-end architecture

(b) Surveillance antennas; (c) Reference antenna.

As shown in Fig. 3 (a), each antenna is connected to a commercial low noise block (LNB), which has the task to amplify the signal in Ku band and perform a down conversion from $\mathrm{Ku}$ to $\mathrm{L}$ band. To keep the four channels phase synchronized, each LNB needs to share through the same connector the direct current (DC), the $10 \mathrm{MHz}$ GPS reference signal and the output of the down conversion. A diplexer circuit is used for each LNB. The $10 \mathrm{MHz}$ reference signal distributed to the four LNBs provides the required coherence among the channels other than implementing a coherent down conversion. The output of the diplexer circuit is sent to a software defined radio (SDR) board that digitizes the signal and transfers the binary data flow to the processing computer.

An acquisition campaign was carried out in October 2020 in Rome, Italy, collecting the DVB-S signals at $10.758 \mathrm{GHz}$, to assess the effectiveness of the proposed scheme. 


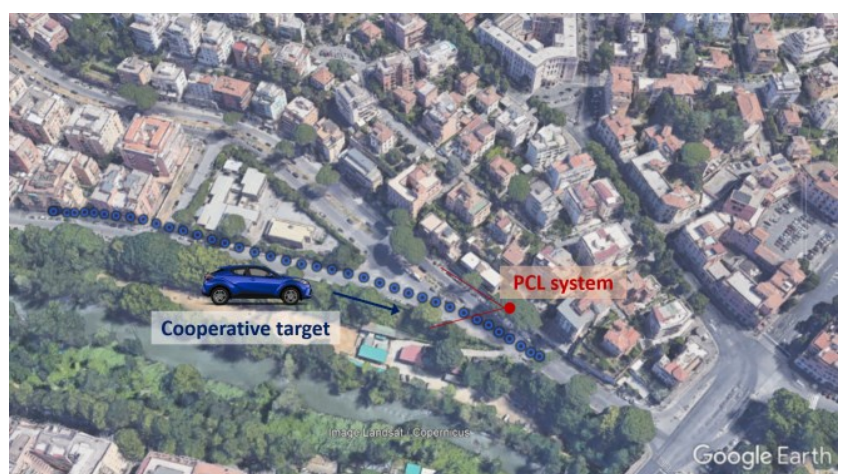

Fig. 4 Acquisition geometry.

The reference antenna is steered towards the satellite EUTELSAT 33E, while the surveillance antennas were steered toward the area to be monitored, $304^{\circ}$ with respect to the north, which includes a road with a cooperative moving target. The acquisition geometry is reported in Fig. 4. Several acquisitions were carried out during the same day and multiple datasets were saved to disk to refine and optimize the processing techniques. During the acquisition used in this work a medium size car (Toyota C-HR) equipped with a GPS was employed as cooperative target (see the blue markers in Fig. 4). Fig. 5 shows its GPS trajectory projected in the bistatic range-velocity plane. The final processing technique were then made ready for real time operation.

\section{EXPERIMENTAL RESULTS}

To show the effectiveness of the system, we selected a dataset with a duration of approx. 28 s processed with the techniques described in Section II, using CPI of $0.5 \mathrm{~s}$. This duration has been selected small enough to enable the real-time processing by guaranteeing two characteristics: (i) limited computational cost for the coherent integration despite the high sampling frequency rate required by the wide signal bandwidth, and (ii) limited target migration effects in range, in Doppler frequency and also in azimuth angle so that advanced (and computationally demanding) processing stages to compensate the migration, [15], don't need to be used.

As a first result, we show in Fig. 5 the PCL detections on the bistatic range-bistatic velocity plane, obtained for all the considered datafiles, overlapped by $0.2 \mathrm{~s}$. The black curve represents the GPS track, the gray dots represent the raw PCL detections, while the red dots represent the PCL detections that have been correctly associated with the GPS trajectory of the cooperative car. By observing Fig. 5, we notice that with the considered processing scheme and the selected parameters, the cooperative car is detected starting from the moment it reaches approx. $220 \mathrm{~m}$ of bistatic range. During the whole acquisition time however, several sequences of continuous plots are visible in the range-Doppler plane. They are likely to correspond to vehicles moving along the same street, which is well within the beamwidth of the surveillance antennas. In fact, various vehicles were present along this street, either heading in the same direction as the cooperating car or in the opposite direction, as well as in the busy neighborhood of the considered area. Among them, we highlight in light blue a sequence of detections that clearly correspond to a medium size motorbike, observed by the cooperating car driver shortly behind him.

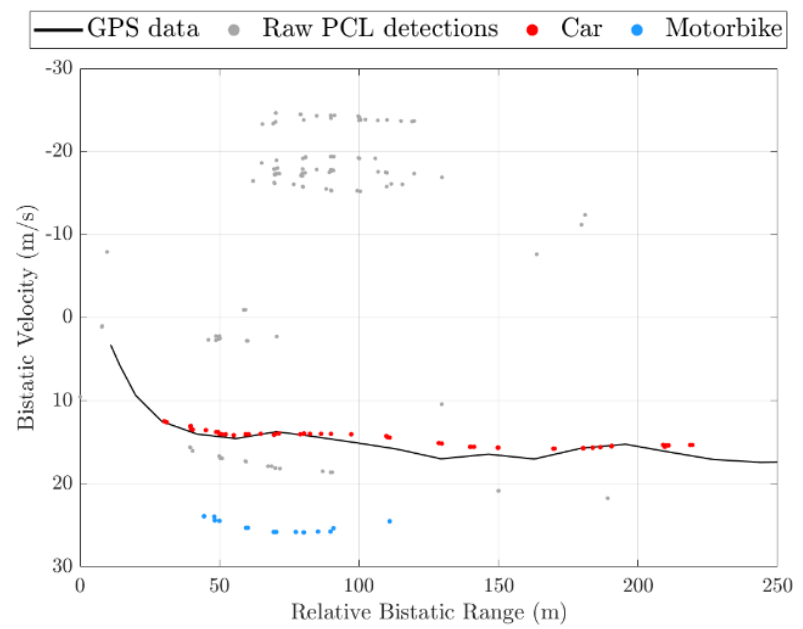

Fig. 5 PCL detections in the bistatic range - velocity plane using $K=3$.

Table II reports the number of correct detections for the two considered targets, when only two of the three surveillance antennas are exploited and for the case where all the three of them are used together to provide the widest unambiguous angle. Table II shows that the use of three antennas yields an increased number of detections of approx. $15 \%$ on the car with respect to the cases with $K=2$. The improvement is less noticeable with respect to the motorbike, which is overall detected with less continuity. This is probably due to the low SNR of its echoes but also to its less controlled motion which could include sudden accelerations that would require an appropriate processing stage to be compensated for.

Fig. 6(a-c) reports the DoA estimated along the acquisition time when only two out of the three available surveillance antennas are used. The dashed lines reported in each figure represent the non-ambiguous angular sector guaranteed with the corresponding two-element antenna. More precisely, the considered antenna configurations correspond to: (a) the left and center antennas in Fig. 3(b), with a distance of $0.1 \mathrm{~m}$, (b) the center and right antennas in Fig. 3 (b) with a distance of $0.15 \mathrm{~m}$ and (c) the left and right antennas in Fig. 3 (b) with a distance of $0.25 \mathrm{~m}$. By observing Fig. 6(a-c), the considerations on Fig. 2 are confirmed, namely (i) the larger is the distance between the antennas (moving from left to right in Fig. 6), the higher is the DoA estimation accuracy, while (ii) the shorter is the distance between the antennas (moving from right to left in Fig. 6), the larger is the angular sector where the DoA is estimated without deterministic ambiguities.

TABLE II NUMBER OF CORRECT DETECTIONS WHEN TWO OR THREE SURVEILLANCE ANTENNAS ARE EMPLOYED.

\begin{tabular}{|c|c|c|c|}
\hline \multirow{2}{*}{\multicolumn{2}{|c|}{ Antenna configuration }} & \multicolumn{2}{|c|}{ Target } \\
\hline & & Car & Motorbike \\
\hline \multirow{3}{*}{$\begin{array}{c}K=2 \\
\text { surveillance } \\
\text { antennas }\end{array}$} & $\mathrm{d}=0.1 \mathrm{~m}$ & 43 & 12 \\
\hline & $\mathrm{d}=0.15 \mathrm{~m}$ & 45 & 11 \\
\hline & $\mathrm{d}=0.25 \mathrm{~m}$ & 44 & 11 \\
\hline \multicolumn{2}{|c|}{$\begin{aligned} K= & 3 \text { surveillance antennas } \\
& \mathbf{d}=\left[\begin{array}{llll}0 & 0.1 & 0.25\end{array}\right] \mathrm{m}\end{aligned}$} & 50 & 13 \\
\hline
\end{tabular}




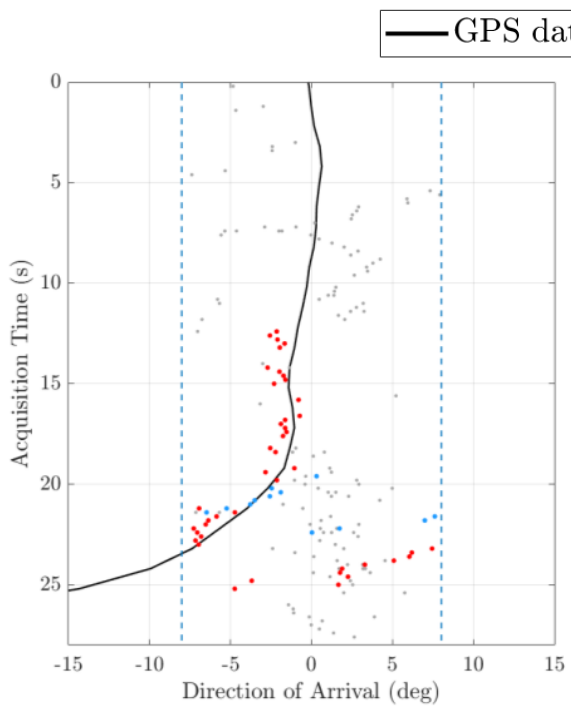

(a)

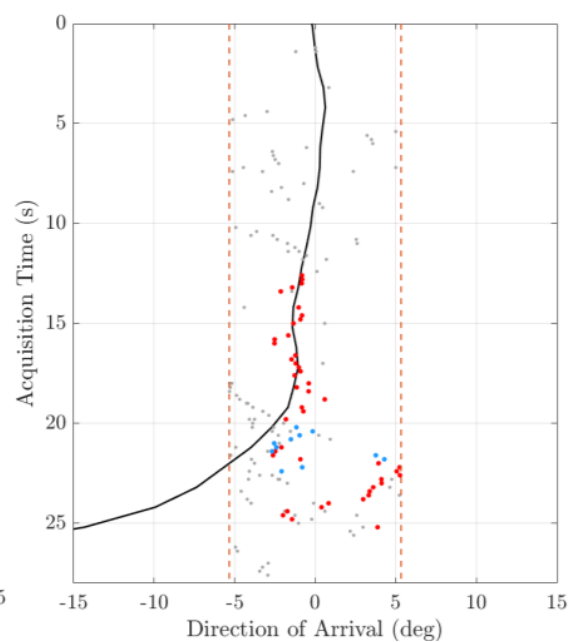

(b)
Motorbike

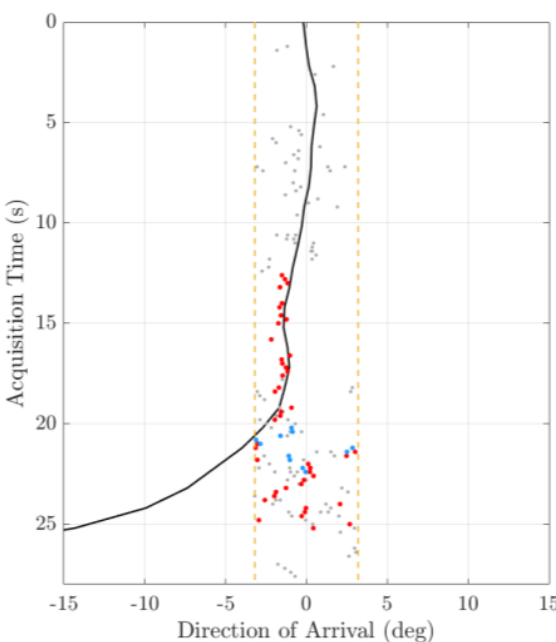

(c)

Fig. 6 Estimated DoA during the acquisition time with $K=2$ surveillance antennas with distance $\mathrm{d}=0.1 \mathrm{~m}$ (a) $\mathrm{d}=0.15 \mathrm{~m}$ (b) and d $=0.25 \mathrm{~m}$ (c).

In fact, car detections on Fig. 6(a) are localized with a poor accuracy along most of the detected portion of the trajectory. On the other hand, car detections in Fig. 6(c) are localized with a better accuracy but only in a very limited angular sector. In all three cases, the motorbike localization is not achieved except for a very short time.

To overcome this issue, Fig. 7 reports the same results obtained using all three available surveillance channels. With the selected configuration, the unambiguous angular sector is well beyond the angular sector of interest. Moreover, we can correctly localize the car almost for the whole portion GPS track and the few plots associated with the motorbike on the range-velocity plane seem to follow a reasonable trajectory.

As apparent, the angular measurement for the car with the three-antenna system appears quite stable during time and only a few significant estimation errors (outliers) are obtained. As previously mentioned, these outliers are likely to be present in correspondence to low target echo SNR values at specific scans. This clearly applies in Fig. 8(a-b) where two range-velocity maps, normalized to the noise floor, are obtained from the first surveillance channel for two different datafiles. In both cases, the highlighted targets are detected and correctly associated to the GPS track on the range-velocity map.

However, there is a difference of approx. $6 \mathrm{~dB}$ between the SNR of the target echo in the two considered scans (see the range and Doppler cross-sections of the two selected targets in Fig. 8(c)), which might be responsible for the occurrence of an outlier. More precisely, the target in Fig. $8(\mathrm{a})$ is wrongly localized (see the red plots at approx. $9^{\circ}$ and 21-22s in Fig. 7) while the target in Fig. 8(b) is correctly localized on the GPS track.

As a final remark, additional peaks are present in the maps in Fig. 8, see e.g. the peak at approx. $-25 \mathrm{~m} / \mathrm{s}$ and $45 \mathrm{~m}$ in Fig. $8(\mathrm{~b})$, which clearly corresponds to the motorbike. It is worth mentioning that the motorbike echo does not appear to be fully focused in the range-velocity plane but has a slightly smeared response, confirming the above hypothesis of a noncompensated target migration effect due to non-controlled vehicle motion. Indeed, the application of advanced migration compensation techniques could provide a fully focused plot in the map, at the expensa of a significant increase in the computational load.

We further observe that both the Range-Velocity maps and the plots in the Cartesian coordinates are reasonably clean, despite the presence of an appreciable number of false alarms that is typical for a passive radar. In particular, it is apparent that there is no significant background structure, see also the cross-section in Fig. 8(c) at the target range cell. This confirms that due to the passive radar geometry obtained for the geostationary IOs, the disturbance cancellation processing technique is not necessary. The validation of the simplified processing scheme, that avoids the cancellation stage and limits the CPI to $0.5 \mathrm{~s}$, allows to obtain a real time operation using a single GPU.

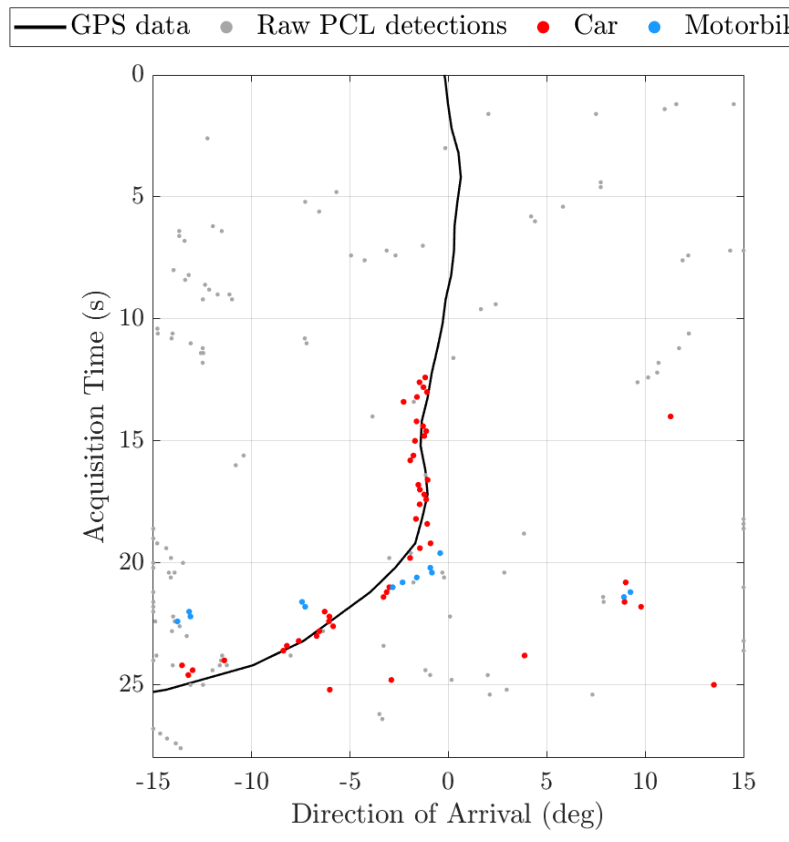

Fig. 7 Estimated DoA during the acquisition time using $K=3$ surveillance antennas 

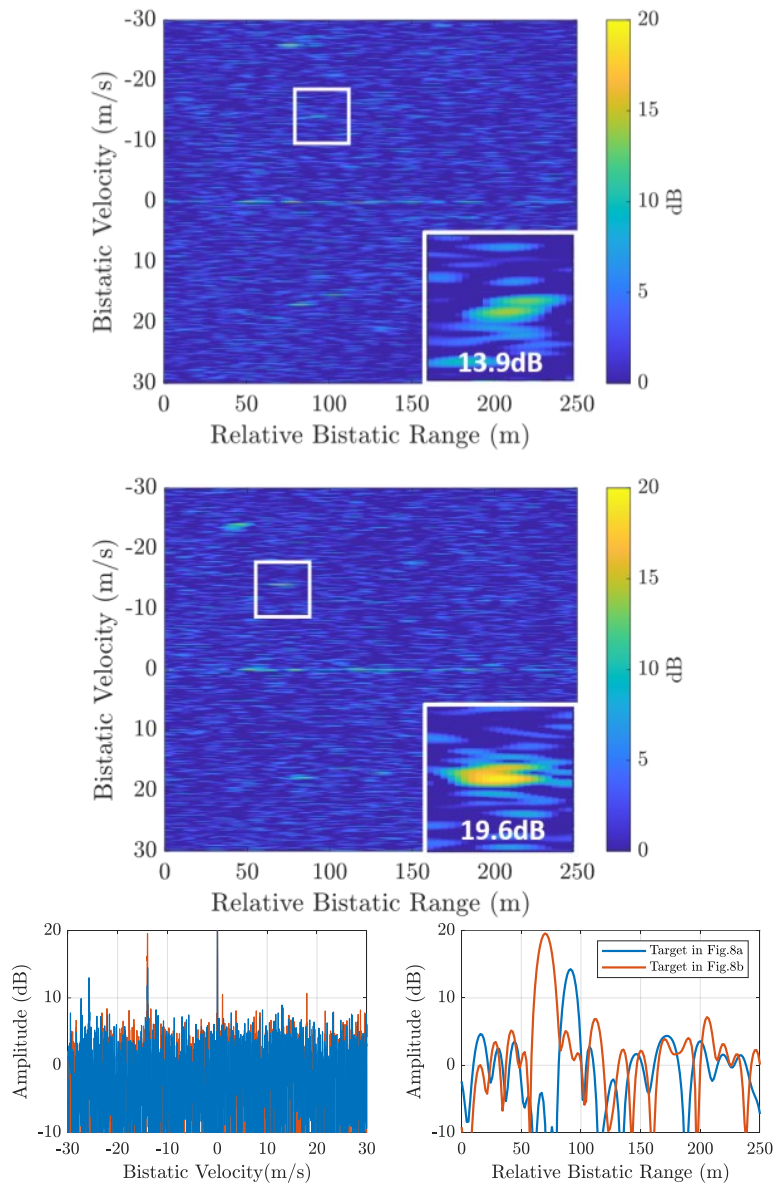

Fig. 8 Range-Velocity maps at two different datafiles and corresponding range and Doppler cross-sections.

\section{CONCLUSION}

In this paper, we have presented the architecture and a lowcost COTS-based implementation of a passive radar system exploiting DVB-S transmissions for short range detection of targets approaching a critical infrastructure to be protected. This includes a high accuracy angular measurement capability, required to complement the high accuracy of its range and velocity measurements, thus providing an accurate localization in the Cartesian plane. Its effectiveness has been demonstrated against a real short-range scenario including a cooperative car. The experiment also validated the presented low-complexity processing scheme, with appropriately selected parameters, that can operate in real-time on a single GPU to detect and localize small and medium-size targets such as cars and motorbikes.

\section{ACKNOWLEDGMENT}

The authors gratefully acknowledge Genex RF s.r.l. for the valuable advices regarding the RF components selection.

\section{REFERENCES}

[1] H. Griffiths and C. J. Baker, An Introduction to Passive Radar. Artech House, 2017.

[2] F. Colone, "Short-range passive radar potentialities" in "Novel Radar Techniques and Applications, Vol. 1", Ch. 15, pp. 661-718.

[3] P. Samczynski, et al., "Passive Radar as a Part of Critical Infrastructure Protection System," 2018 International Conference on Radar, Brisbane, QLD, 2018, pp. 1-5.
[4] Y Liu, et al., "Digital television based passive bistatic radar system for drone detection," 2017 IEEE Radar Conference, Seattle, WA, May 2017, pp. 1493-1497.

[5] C. Schüpbach, et al., "Micro-UAV detection using DAB-based passive radar," 2017 IEEE RadarConf, Seattle, WA, pp. 10371040.

[6] D. Poullin, "Countering illegal UAV flights: passive DVB radar potentiality," 19th Intern. Radar Symp., Bonn, 2018, pp. $1-10$.

[7] N. del-Rey-Maestre, et al. "Optimum Beamforming to Improve UAV's detectoon Using DVB-T Passive Radars," 2019 IEEE International Radar Conference, Toulon, France, Sept. 2019.

[8] T. Martelli, F. Colone and R. Cardinali, "DVB-T based Passive Radar for simultaneous counter drone operations and civil air traffic surveillance," IET RSN, vol. 14, no. 4, pp. 505-515, 4 2020.

[9] B. Knoedler, R. Zemmari, and W. Koch, "On the detection of small uav using a gsm passive coherent location system, " 2016, vol. 2016-, IEEE Computer Society.

[10] P. Falcone, et al. "Two-dimensional location of moving targets within local areas using WiFi-based multistatic passive radar," in IET RSN, vol. 8, no. 2, pp. 123-131, Feb. 2014.

[11] C. Ilioudis, C. Clemente and J. Soraghan, "GNSS-based passive UAV monitoring: a feasibility study," in IET RSN, vol. 14, no. 4, pp. 516-524, 42020.

[12] P. Gomez-del-Hoyo et al., "First Approach on Ground Target Detection with GPS based Passive Radar: Experimental Results," 2019 Signal Processing Symposium (SPSympo), Krakow, Poland, 2019, pp. 71-75,

[13] O. Cabrera, et al., "Detecting drones and human beings with DVB-S based COTS passive radar for short-range surveillance," 2020 IEEE Int. Radar Conference, Washington, DC, USA, 2020, pp. 37-42.

[14] M. Ummenhofer, et al., "UAV Micro-Doppler Signature Analysis Using DVB-S Based Passive Radar," 2020 IEEE International Radar Conference, Washington, DC, USA, 2020, pp. 1007-1012.

[15] T.Martelli, O.Cabrera, F. Colone and P. Lombardo, " Exploitation of long coherent integration times to improve drone detection in DVB-S based Passive Radar," 2020 IEEE Radar Conference, Florence, Italy, 2020.

[16] F.Colone, et al.: "A multistage processing algorithm for disturbance removal and target detection in passive bistatic radar", IEEE Transactions on Aerospace and Electronic Systems, vol. 45, no. 2, Apr. 2009.

[17] P. Lombardo, and F. Colone, "Advanced processing methods for passive bistatic radar systems," in Principles of Modern Radar: Advanced Radar Techniques, W. L. Melvin, and J. A. Scheer, Raleigh, NC: SciTech Publishing, 2012.

[18] M. Viberg, B. Ottersten and A. Nehorai, "Performance analysis of direction finding with large arrays and finite data," in IEEE Trans. on Signal Process, vol. 43, no. 2, pp. 469-477, Feb. 1995.

[19] R. L. Goodwin, "Ambiguity-resistant three-and four-channel interferometers," NRL Report 8005, Naval Research Laboratory, Washington, D.C. 20375, September 1976.

[20] F. Athley, "Threshold region performance of maximum likelihood direction of arrival estimators", IEEE Trans. Signal Process., vol. 53, no. 4, pp. 1359-1373, Apr. 2005.

[21] F. Filippini, F. Colone and A. De Maio, "Threshold Region Performance of Multicarrier Maximum Likelihood Direction of Arrival Estimator," in IEEE Transactions on Aerospace and Electronic Systems, vol. 55, no. 6, pp. 3517-3530, Dec. 2019. 